\title{
Une façon platonicienne d'être une image: le mimema*
}

\author{
MARIE-LAURENCE DESCLOS
}

Grenoble 2

\begin{abstract}
RESUMO: O artigo faz uma reflexão sobre o estatuto ontológico da imagem enquanto resultado, compreendido como mímema, do processo produtivo que é a atividade demiúrgica, compreendida como mímesis. A noção de mímema é analisada em passagens de Ésquilo e de Eurípides e compreendida como a transcrição, mais que a reprodução, de um modelo sobre um outro suporte. Ao estudar o contexto de utilização do termo mímema pelos poetas, a autora ilumina justamente aquilo que será conceptualizado por Platão em sua teoria da mímesis.
\end{abstract}

PALAVRAS-CHAVE: mímema; mímesis; Platão.

Assez curieusement, c'est le constat d'une absence qui a suscité les quelques remarques que je me propose de présenter dans les lignes qui suivent. Tenant, comme tant d'autres, qu'il n'est guère possible de parler d' "images" chez Platon sans prendre en compte ce qu'il est convenu d'appeler sa théorie de la mimesis, je me suis avisée que - dans le récit consacré à la fabrication du monde par le Démiurge - le verbe $\mu \iota \mu \varepsilon \hat{\imath} \sigma \theta \alpha \iota$ n'apparaissait jamais. Étrange absence, si l'on veut bien se souvenir que, dans le Timée, c'est l'exercice d'un regard fixé sur le modèle qui fonde l'activité démiurgique ${ }^{1}$. Exercice d'un regard et non imitation. Il n'en reste pas moins que $\pi \alpha \rho \alpha ́ \delta \varepsilon \imath \gamma \mu \alpha$ et $\beta \lambda \varepsilon ́ \pi \varepsilon \iota v$ appartiennent au champ sémantique de la $\mu$ í $\eta_{\eta} \sigma \varsigma$. Victor Goldschmidt le soulignait: $\beta \lambda \varepsilon ́ \pi \varepsilon \imath \nu$, avec ou sans préverbe, sert de façon habituelle à "indiquer que l'on s'inspire d'une forme-paradigme, soit [...] pour définir, soit pour agir"’2. On pourrait m'objecter que s'inspirer n'est pas imiter. Bien qu'il ne soit plus besoin d'insister sur les liens qui unissent paradigme et imitation ${ }^{3}$, je rappellerai cependant République x, 595 c: "L'imitation, d'une façon générale, serais-tu à même de me dire ce qu'elle peut bien être?" Le développement bien connu sur les trois sortes de lit est introduit par cette question. Socrate y déclare, parlant de l'artisan qui fabri-

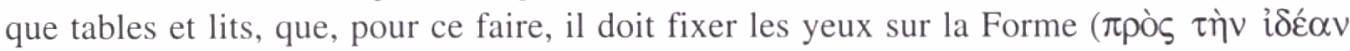

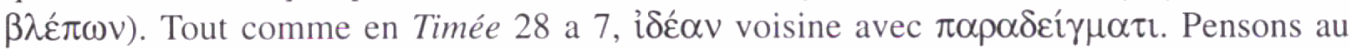
Phèdre, également, où le discours de Lysias fait figure d'anti-paradigme, de paradigme

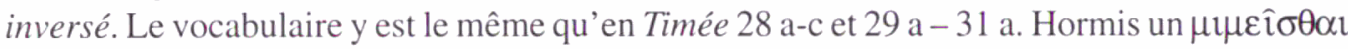
en plus, ce qui n'enlève rien, bien au contraire, à ma démonstration: le discours de Phèdre est saturé de $\pi \alpha \rho \alpha \delta \varepsilon i ́ \gamma \mu \alpha \tau \alpha$ sur lesquels on aurait profit à fixer les yeux [ $\beta \lambda \varepsilon ́ \pi \varepsilon \iota \nu \pi \rho o ́ \varsigma]$ en 
essayant de ne pas les imiter [ $\mu \iota \mu \varepsilon i ́ \sigma \theta \alpha 1](P h d r 264$ a). Continuons nos lectures. Le Timée, en 39 e, est sans ambiguïté: si les astres parcourant le ciel ont été engendrés, "c'est pour que ce monde-ci ressemble le plus possible (óhoró $\alpha \alpha \tau \mathrm{v})$ au vivant parfait et intelligible et pour qu'il imite sa nature éternelle". Je ne reviendrai pas sur les rapports qu'entretiennent

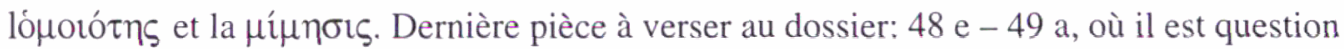

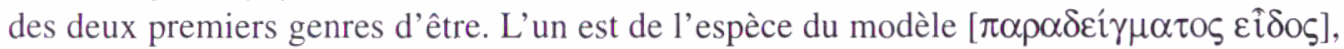
intelligible et immuable, le second est une imitation du modèle [ $\mu\{1 \mu \eta \mu \alpha \pi \alpha \rho \alpha \delta \varepsilon i \gamma \mu \alpha \tau \sigma \varsigma]$,

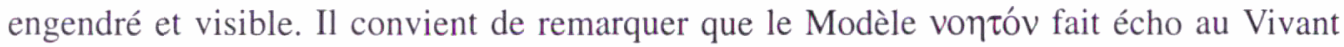
intelligible $(\operatorname{vo\eta } \tau \hat{\omega})$ de 39 e 1-2, tout comme son immutabilité en rappelle la "nature éternelle". Tout semble donc indiquer que la démiurgie divine est, fondamentalement, une activité mimétique.

Faut-il donc se préoccuper de l'absence du verbe $\mu \imath \mu \varepsilon \hat{\imath} \sigma \theta \alpha$ l lors même que $\mu i ́ \mu \eta \mu \alpha$ et $\mu$ í $\eta\rceil \sigma \zeta$ concourent à caractériser un type d'activité dont j'ai par ailleurs montré qu'il était d'entrée de jeu à situer dans le champ sémantique de l'imitation? Le Sophiste semble bien nous rappeler à l'ordre: "nous appelons verbe ce qui rend manifestes les actions" (262 a). Est-ce à dire que l'action démiurgique se révèlerait ainsi étrangère à toute mimétique? Est-ce pour cette raison - le nom étant "le signe vocal qui est appliqué à ceux qui produisent les actions" - que, parmi les nombreux ỏvó $\mu \alpha \tau \alpha$ qualifiant le Démiurge, aucun ne le désigne comme "imitateur"? Mais alors, comment est-on passé du Modèle-à-imiter à l'imageimitée du Modèle? Quel est, enfin, le statut ontologique de cette image-imitée?

On doit tout d'abord prendre acte de l'image qu'utilise Timée pour donner à penser la génération du Monde ${ }^{4}$. Cette image est celle de l'activité démiurgique et, plus encore, de la polytechnie démiurgique. Il y a là, sans nul doute, volonté de faire-comprendre, nécessité de rendre accessible ce qui, par nature, est étranger au monde sensible (Brisson, 1994, p. 83). On peut même, lorsqu'il s'agit d'expliquer le choix de cette image plutôt que de toute autre, l'interpréter comme un des affleurements de ce "système dissimulé [...] qui fait de la fonction artisanale [...] le centre de l'activité humaine, l'activité par excellence" (Vidal-Naquet, 1981, p. 308). Mais est-il possible de faire ce choix singulier dans le chœur des cosmologies grecques sans prendre en compte ce qui donne à la démiurgie sa dimension essentielle, et sans en tirer parti (Brisson, 1974, p. 84-86)? Or ce qui, fondamentalement,

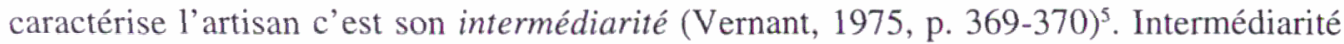
entre une forme et une matière à informer, entre un $\varepsilon \hat{i} \delta$ o $\zeta$ et l'objet dans lequel il va trouver à s'incarner, entre l'usager et la satisfaction de son besoin. Intermédiarité qui rend possible le passage d'un modèle à un produit par définition extérieur à l'activité de celui qui le fabrique. Intermédiarité qui n'est pas véritablement "action" mais bien plutôt "opération d'ordre instrumental” (Vernant, $1974^{2}$, p. 35). La différence, en effet, n'est pas grande entre la tarière qui sert à percer, et le forgeron qui sert à imposer au fer la forme de la tarière. L'un et l'autre ne sont, en denière analyse, que les instruments dont se servira ( $\chi \rho \eta ́ \sigma \varepsilon \tau \alpha \mathrm{l})$ le perceur (Crat. 388 a - 389 c). Le Démiurge n'imite pas à proprement parler: il ne fait que rendre possible l'effectuation d'un processus d'imitation. Le texte d'ailleurs, sur ce point, est fort clair. Que l'on relise, pour s'en persuader, la phrase conclusive du développement sur les astres errants (Tim. 39 d 7 - e 2). Le Démiurge n'y est pas en position de sujet. Le

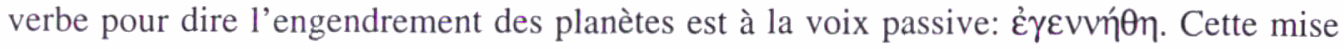
entre parenthèses du sujet de l'action a pour corollaire l'objectivation du "produit" que 
l'aoriste pose comme du déjà-là, du déjà-créé, doté par conséquent d'une existence autonome par rapport à son "producteur" (Vernant, $1974^{2}$, p. 41 ). Toutes choses que vient

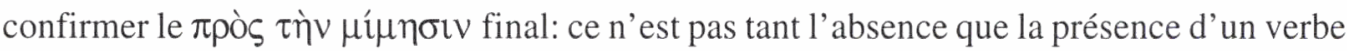
$\mu \iota \mu \varepsilon \hat{\imath} \sigma \theta \alpha$ qui eût été étonnante. Pas de verbe, en effet, mais un de ces mots en - $\sigma \varsigma \varsigma$ que Benveniste caractérisait comme "essentiellement propres à indiquer des opérations objectivées et retranchées de tout rapport avec l'opérateur", exprimant "la notion comme étant hors du sujet et, en ce sens, objective, et posée comme accomplie du fait qu'elle est objective" (Benveniste, 1975, p. 82, 86). Notons que ce processus d'effacement est rendu nécessaire par la nature du Modèle que contemple l'Ouvrier divin, comme l'ont montré les pertinentes analyses de Victor Goldschmidt: "la causalité est fonction du modèle; l'ouvrier se borne à traduire le modèle dont l'action causale agit comme par son intermédiaire" (Goldschmidt, 1970, p. 98 n. 169).

Cette "action" a un résultat: le Devenir est le $\mu$ í $\mu \eta \mu \alpha$ de l'Être. Ce qui est une façon d'affirmer l'extrême ressemblance, une "ressemblance à la Dédale" ( Mí $\mu \eta \mu \alpha$ si fidèle qu'il fait dire au chœur des Satyres dans les Théores d'Eschyle:

Ma mère aurait grand embarras.

A le voir, certainement,

Elle s'enfuirait en criant,

Croyant que c'est moi, celui

Qu'elle a nourri, tellement c'est mon portrait craché.

Si fidèle, qu'il abuse Teucer et Ménèlas: Hélène n'est plus que la copie d'Hélène $\left(\mu\left\{\mu \eta \mu \text { ' 'E } \lambda \dot{\varepsilon ́}^{\prime} \eta \varsigma\right)^{7}\right.$. Nous sommes loin pourtant, comme on va le voir, du "sosie" de Cratyle. Car le $\mu i ́ \mu \eta \mu \alpha$ dit aussi la dissemblance: par rapport au modèle, il pèche par défaut. Au $\mu \dot{t} \mu \eta \mu \alpha$ des Satyres, il manque la parole, et c'est un corps de chair dont - comble du paradoxe - est dépourvu le $\mu$ í $\mu \eta \mu \alpha$ d'Hélène ${ }^{8}$.

Extrême ressemblance, inévitable écart: la copie ne doit jamais être le double parfait du modèle, et elle ne le peut pas plus qu'elle ne le doit, comme en témoignent les textes, peu nombreux, où l'on trouve des attestations de $\mu$ í $\mu \eta \mu \alpha$.

\section{Eschyle}

* Les Théores, Papyrus d'Oxyrrinque (vol. xviII, n² 2162):

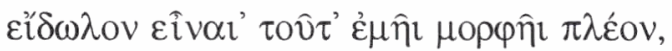

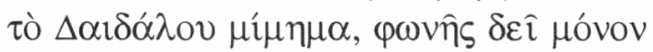

Regarde si cette image pourrait ressembler d'avantage à mon apparence,

Cette copie à la Dédale; il ne lui manque que la parole.

* Frgt n 364 (Nauck), Tragicorum Graecorum Fragmenta:

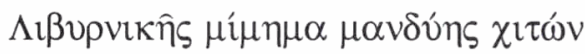

Un chiton, copie d'un manteau liburnien. 


\section{Euripide}

* Héraclès furieux, v. 294:

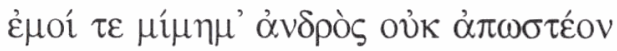

Il ne me faut pas repousser au loin la copie de mon homme.

* Héraclès furieux, v. 992:

$\mu \nu \delta$ рокти́лоv $\mu$ í $\mu \eta \mu \alpha$

(Héraclès devant son fils) copie de celui qui bat le fer rouge.

* Ion, v. 1429:

$\delta \rho \alpha ́ \kappa о \nu \tau \varepsilon \varsigma[\ldots] \pi \alpha \gamma \chi \rho \cup ́ \sigma \omega \ldots$

[...]

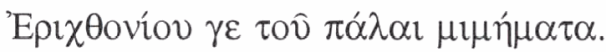

Des serpents; $[\ldots]$ tout en or...

[...]

copies d'Erichtonios l'ancien.

* Troyennes, v. 922 :

$[\ldots] \beta \rho \varepsilon ́ \varphi \circ$,

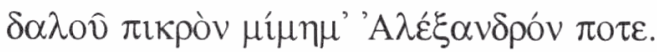

[...] le bébé,

qui sera un jour Alexandre, cette amère copie d'une torche.

* Hélène, v. 74:

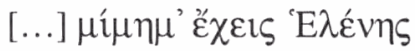

(Teucer à Hélène) tu portes la copie d'Hélène.

* Hélène, v. 875:

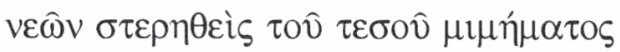

(Ménélas) séparé de ses vaisseaux et de ta copie.

* Frgt n ${ }^{\circ} 25$ (Nauck), Tragicorum Graecorum Fragmenta:

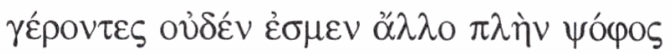

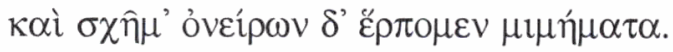

Nous les vieillards ne sommes rien d'autre que vain bruit et simple apparence, copies de songes nous avançons lentement. 
Premier constat, à la lecture de ces quelques vers: le $\mu i ́ \mu \eta \mu \alpha$ est toujours déceptif. Voilà qui ne fait aucun doute pour quatre des neuf attestations: le fragment des Théores, les vers 74 et 875 de l'Hélène, et le fragment 25 (Nauck). En l'absence de tout contexte, on ne peut juger du second fragment d'Eschyle. Reste cependant à savoir qui est abusé dans les quatre dernières occurrences.

Si le $\mu$ í $\mu \eta \mu \alpha$ est déceptif, c'est parce qu'il y a ressemblance et, on la vu, ressemblance extrême. Mais le $\mu$ í $\mu \eta \mu \alpha$ c'est aussi, c'est surtout la dissemblance par rapport au modèle, qui le distingue du sosie. Cette dissemblance peut n'être pas perçue: "ma mère aurait grand embarras à le voir $[\ldots]$ croyant que c'est moi". Il ne naît alors, pour qui s'est laissé prendre au piège, qu'inquiétants quiproquos. Il en va tout autrement lorsque la dissemblance est d'entrée de jeu posée et connue: la déceptivité du $\mu$ í $\mu \eta \alpha$ est comme montrée du doigt tout en étant, dans le même temps, revendiquée et assumée, ce qui lui donne tout son poids. Dire d'Héraclès qu'il est "copie de celui qui bat le fer rouge", c'est affirmer tout à la fois que rien ne l'en distingue et que tout l'en sépare. Dans le jeu entre ce rien et ce tout "il y a la tragédie". Tout

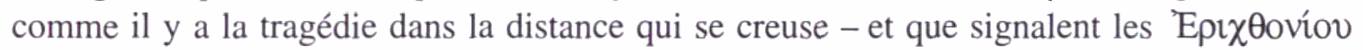
$\mu \tau \mu \eta \dot{\mu} \mu \tau \alpha-$ entre Ion et Erichthonios: "les serpents protecteurs se sont mués en bijoux d'or, parure de deuil" (Loraux, 1981, p. 209). Ici le $\mu$ í $\mu \mu \alpha$ répète, mais en l'inversant, la naissance de l'autochtone. C'est le même procédé qui est à l'œuvre dans le vers 922 des Troyennes. Marie Delcourt semble fidèle à la tradition, et à Euripide ${ }^{9}$, lorsqu'elle traduit:

"[...] le nouveau-né,

figuré par la torche néfaste du rêve,

l'enfant qui serait un jour Alexandre."

Sa traduction, cependant, atténue singulièrement le sens et la portée de la phrase grecque. Car, si le propos d'Hélène répète le songe d'Hécube, cette répétition n'est pas répétition à l'identique mais, là encore, répétition inversée. La torche du songe disait à Hécube la vérité de l'enfant à naître tandis qu'Hélène révèle la torche sous le masque enga-

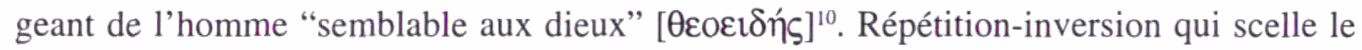
destin de Pâris-Alexandre. De fait, entre la torche dont rêve Hécube et celle dont parle Hélène, il y a l'intervalle qui distingue tout ce qui doit encore être accompli de tout ce qui ne peut plus être évité; il y a ce que dévoile le songe prémonitoire, et ce que dissimule une copie déceptive. Bien que tout l'en sépare, rien ne distingue Héraclès du $\mu \nu \delta \rho \circ \tau \tau u ́ \pi o v ;$ tout distingue Alexandre du sinistre brandon, mais rien ne l'en sépare. L'écart, contrairement à l'usage habituel de $\mu$ í $\mu \eta \mu \alpha$, est un écart de "forme extérieure": rien, chez Pâris, ne dit la torche. Si la copie est déceptive, c'est en raison de sa non-ressemblance, c'est-à-dire d'une déceptivité autre ou, plus exactement, d'une déceptivité poussée à ses limites. Jusqu'au point où même le rapport entre ressemblance et écart s'inverse: ce qui ressemble n'est pas ce que l'on voit, parce que la ressemblance se situe à un autre niveau, sur un autre plan. Le $\mu$ í $\mu \eta \mu \alpha$ ne peut donc se donner pour ce qu'il est. Dès lors, soutenir qu'Alexandre est $\delta \alpha \lambda \mathrm{ov} . . . \mu$ í $\mu \eta \mu \alpha$ revient à restituer à la torche son statut de modèle, à faire coexister les deux ordres, les deux plans le temps d'une phrase, à faire affleurer ce qui d'ordinaire ne se voit pas. La déceptivité, ici, ne naît pas d'une trop grande similitude entre la copie et son modèle, mais de la dissimulation du modèle. Ce que dit, plus métaphoriquement, l'enlève- 
ment d'Hélène par le fils de Maia, et son enfermement au pays de Protée: elle aussi devra rester cachée.

Pour Mégara, également, la ressemblance se situe dans un registre différent. Face à la menace que Lycos, meurtrier de son père et usurpateur du trône, fait peser sur elle, sur ses enfants et sur Amphitryon, l'épouse d'Héraclès déclare: "il ne faut pas repousser au loin la

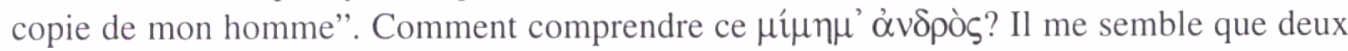
hypothèses, qui ne sont pas forcément exclusives l'une de l'autre, peuvent être avancées. Il convient tout d'abord de constater que $\mu i \tilde{\mu} \mu \alpha \alpha$ évolue dans le champ sémantique des

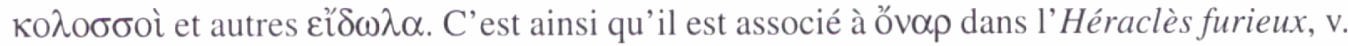

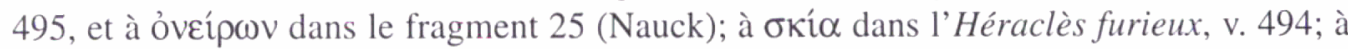

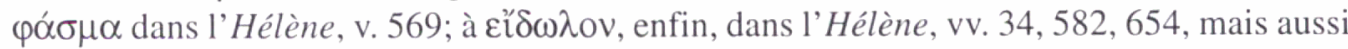
dans l'Electre, v. 1282, et dans les Théores. On peut donc sans grand risque ranger le $\mu$ í $\mu \eta \alpha$ dans ce que Jean-Pierre Vernant appelle la "catégorie du double" (Vernant, 1974, p. 69-70). Mais peut-on aller plus loin? Peut-on rapprocher le $\mu$ í $\mu \eta \mu \alpha$ d'Héraclès de ces "figurines de remplacement" qui ornent le palais de Ménélas, et qui sont "utilisées par la magie amoureuse pour évoquer l'absent comme elles le sont, dans les rites funéraires, pour évoquer le mort" (Vernant, 1974, p. 71)? Hypothèse séduisante, si l'on veut bien se souvenir qu'à Thèbes c'est un mort que l'on pleure en pleurant Héraclès. Le désir de l'absente qui trouble Ménélas (Agamemnon, v. 414: $\pi$ ó $\omega$ ) trouve son parallèle dans le désir de l'absent éprouvé par le chœur (Héraclès furieux, v. 269: $\pi$ óӨov). On pourrait m’objecter que, sur cette "figurine de remplacement", on ne trouve aucune indication, qu'il n'en est fait aucune description. Et, si rien dans le texte ne vient infirmer mon hypothèse, rien, semble-t-il, ne vient non plus la confirmer. Sauf à considérer le discours du couple Lycos-Amphitryon ", et ce qu'Arthur S. Way appelle "le lai des travaux d'Hercule", comme la plus belle des descriptions. De

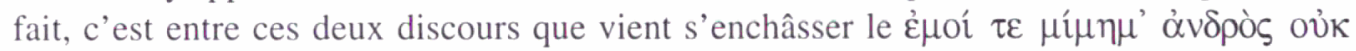

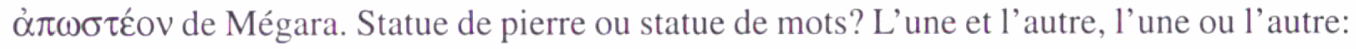

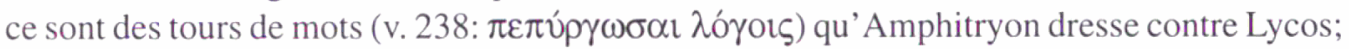
c'est un $\alpha \not \gamma \alpha \lambda \mu \alpha$ de discours que le chœur veut élever pour Héraclès (v. 358). Dans cette tragédie, les mots valent la pierre et les discours valent statues. Dès lors, ne pas repousser au loin le $\mu$ í $\uparrow \mu \alpha$ d'Héraclès, n'est-ce pas une façon de maintenir "la liaison des vivants avec le monde infernal" par ailleurs si souvent évoqué (Vernant, 1974, p. 73) ${ }^{12}$ ? N'est-ce pas une façon d'assurer la présence du héros malgré son irréparable absence? On remarquera enfin que le $\mu$ í $\mu \eta \alpha \alpha$, dans l'usage que semble en faire ici Euripide, ne se borne pas à n'être qu'un terme "plus technique [...] désignant l'image dans sa fonction de ressemblance concertée à un modèle"; il peut aussi être porteur des valeurs qui, dans la pensée archaïque, étaient celles du double: "ressemblance [...] de l'altérité radicale de [l']être actuel de mort, de [...] son exil en un ailleurs où s'inversent toutes les réalités d'ici-bas" (Vernant, 1990, p. 29 et 37). Rien n'empêchera donc les "images" platoniciennes, qui sont autant de $\mu \imath \mu \eta$ ' $\mu \alpha \tau \alpha$, d'en être, elles aussi porteuses - ou, à tout le moins, certaines d'entre elles.

Une autre interprétation peut être avancée, qui ne fait pas du $\mu$ í $\mu \eta \mu \alpha$ une manière de

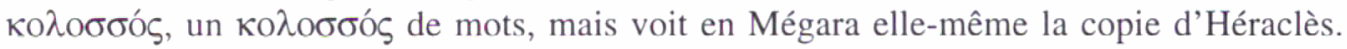
Curieuse "copie", cependant, et curieuse façon de le devenir. Jugeons-en plutôt. Mégara craint la mort, mais accepte de mourir (vv. 281-283). Est-ce là courage digne d'Héraclès? La résignation n'est-elle pas au plus loin de cet "effort pénible" ( $\pi$ óvov, v. 89) qu'incarne 


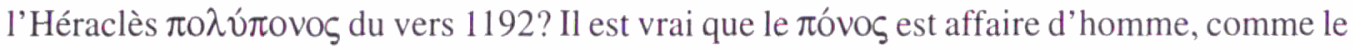

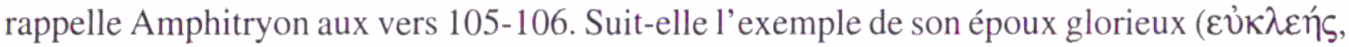
vv. 288 et 290) lorsqu'elle préfère pour ses enfants la mort à une vile renommée ( $\delta$ ó $\xi \alpha \mathrm{V}$

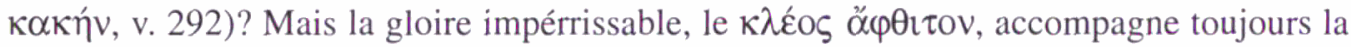

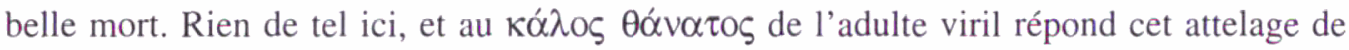
morts, composé de ceux qui, par leur âge ou par leur nature, sont exclus des champs de

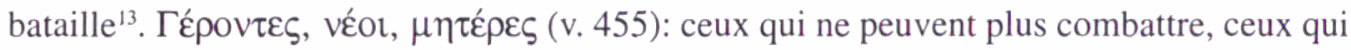
ne le peuvent pas encore et celles qui, en raison de leur sexe, sont exclues des travaux d'Arès. La mort choisie par Mégara - acceptation du meurtre, du sacrifice impie - ne peut susciter l'éloge réservé au guerrier valeureux ${ }^{14}$, tout au plus pourra-t-elle éviter le rire (v. 285 ) dont on sait qu'il est proche parent de la honte ${ }^{15}$. Au bout du compte, peu importe, car

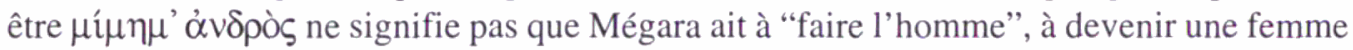
déviante, une seconde Clytemnestre, mais à transcrire le modèle de l'ỏví $\rho$ sur ce support autre qu'est, pour l'épouse d'Héraclès, son être-femme. Et ce "support" exige que la mort au combat se fasse suicide ou sacrifice: il en sera ainsi puisqu' aussi bien le meurtre n'est ici - comme ailleurs, souvent - qu'un suicide forcé ou un sacrifice corrompu ${ }^{16}$.

On est donc en présence d'un jeu sur l'altérité et l'identité, mais aussi sur la présence et l'absence, la répétition et l'inversion, le montré et le caché, la ressemblance et l'écart. A l'origine de ce jeu la transcription d'une réalité sur un support autre.

Le $\mu$ í $\mu \eta \mu \alpha$ se situe donc sur un autre registre que ce dont il est la copie, par quoi il faut comprendre non pas tant l'écart entre la réalité et son image, que la dissimilitude de leur substrat, ou, pour parler plus précisément, l'écart entre la réalité et son image trouve son fondement dans la dissimilitude du substrat. En d'autres termes, l'être du $\mu$ í $\mu \eta \mu \alpha$, consiste moins en la reproduction de la réalité par l'image, qu'en la transcription de cette réalité sur un support autre. Ce qui, d'entrée de jeu, interdit toute stricte duplication du modèle. Par "support autre" il faut entendre "cause matérielle" autre: masques de terre cuite, serpents d'or, corps d'éther, homme de pierre ou de mots, courage de femme ${ }^{17}$. Ce changement de "support" ne se contente pas cependant d'exprimer une différence de niveau de réalité: il la fait venir à l'être. Qu'il me soit permis, pour justifier mon propos, de m'arrêter un instant sur le vers 992 de l'Héraclès furieux. Voici Lyssa gravant sur le corps du héros la mania voulue par Héra (vv. 867-871). Mais, pour être $\mu \alpha$ ıvó $\mu \varepsilon v o \zeta$, Héraclès n'en reste pas moins ce qu'il est. Possédé, il le sera, mais à sa façon. Dès lors faut-il s'étonner si, à la semblance d'Héphaistos qui, en haut de l'Etna, forge ( $\mu \nu \delta \rho \circ \kappa \tau v \pi \varepsilon \hat{\imath}$, Prométhée enchaîné, v. 366), le fils du Cronide devient "copie de celui qui bat le fer rouge", $\mu v \delta \rho \circ \kappa \tau v ́ \pi o v$ $\mu \prime \mu \eta \mu \alpha$ ? Les liens, en effet, sont étroits entre le dieu forgeron et le héros à la peau de lion. La transcription, pour opérer, n'a qu'à inverser les signes: l'illustre Boiteux ouvrait la cuirasse et l'écu protecteurs, alors qu'Héraclès "forge" la destruction de sa propre maison. Inversion des signes, également, dans le fonctionnement déréglé de cette magie guerrière dont il est tout environné, et qu'il ne parvient plus à maîtriser ${ }^{19}$. Il y a identification du héros au monstre dont il porte, sur son bouclier, le masque grimaçant. Celui dont les yeux ont le mortel éclat des yeux de Gorgo (Bouclier, vv. 223-224; Héraclès furieux, v. 990), celui qui peut tout à la fois susciter l'Effroi, le terrible Phobos (vv. 950, 971), et vaincre la Terreur, le Deimos redoutable (v. 700), s'est fait vivante égide ${ }^{19}$. Voué comme Créuse à Nuit la ténébreuse (vv. 822, 883), comme Créuse "terrifiant et gorgonesque" (vv. 868, 890), il ne peut - 
pas plus que Créuse - dominer le pouvoir maléfique de la magie gorgonéenne. Nicole Loraux a pu dire de Créuse qu'elle avait choisi "le côté de Pallas [...] magicienne et guerrière" (Loraux, 1981 , p. 244) ${ }^{20}$. Héraclès, quant à lui, s'y trouve précipité. Créuse n'a fait que frôler la catastrophe, le fils d'Alcmène n'a pas pu l'éviter. De Pallas, d'ailleurs, il faudra l'intervention -

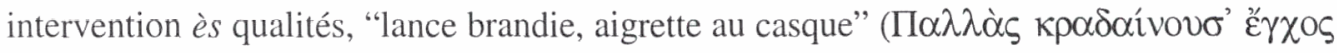

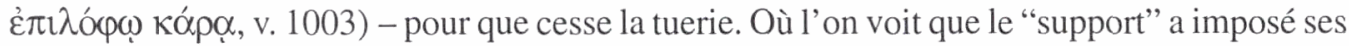
propres exigences à ce dont il devait porter la marque. En d'autres termes, le support compte autant que le modèle, et c'est bien pourquoi la mania du héros ne pouvait être qu'une furie guerrière s'accompagnant du retournement des forces, hier protectrices ou bienveillantes.

Etre un $\mu$ í $\mu \eta \alpha \alpha$ c'est donc être le résultat d'un jeu très subtil qui se joue entre la

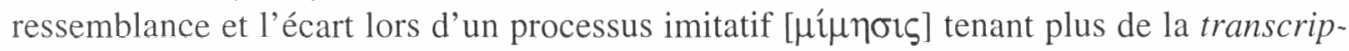
tion que de la banale reproduction. Ce qui le distingue de la copie-conforme, ou du sosie. Il est cependant déceptif lorsque seule la ressemblance est retenue - lorsqu'il abuse celui qui le contemple -, mais aussi quand l'écart est perçu: d’une déceptivité revendiquée qui lui donne sa véritable dimension. Ce n'est plus alors un simple leurre fauteur de quiproquos, mais une notion mère d'ambiguïté qui me semble ne pouvoir être mieux exprimée que par une définition en forme de paradoxe: le $\mu$ í $\mu \eta \mu \alpha$, un écart-ressemblant engendré par la transcription d'un modèle sur un support dont ils diffèrent. Tel est le contexte d'utilisation dans lequel Platon, en le conceptualisant, va inscrire sa propre théorie de la $\mu$ í $\mu$ ¡ıs Elle lui permettra tout à la fois de préciser la nature et la fonction de l'image, et de sauvegarder la nécessaire distinction entre l'Être et le Devenir, le Paraître et l'Apparaître.

J'ai précédemment tenté de montrer en quoi la démiurgie était, par l'exercice d'un regard, activité mimétique. Cette activité, par ailleurs, est à placer sous le signe de l'intermédiarité: intermédiarité d'une démiurgie mimétique entre un modèle et sa copie, entre deux niveaux de réalité différents. Rappelons-nous ce que déclarait Victor Goldschmidt: "L'imitation n'est pas un calque. C'est la reproduction d'un modèle dans un ordre différent"(Goldschmidt, 1970, p. 99). L'analyse du $\mu$ í $\mu \eta \mu \alpha$ dans l'usage qu'en fit la tradition me semble avoir permis tout à la fois de confirmer et de préciser cette interprétation. De la confirmer, car il y a bien passage d'un ordre à un autre; de la préciser, car ce passage est plus transcription que reproduction: non pas reproduction, ou même transposition (Saïd, 1987, p. 311) de l'Être dans le Devenir, mais transcription de l'Être sur un support autre, tel qu'un $\mu i ́ \mu \eta \mu \alpha$ de l'Être - le Devenir - puisse y apparaître. Peut-être aura-t-on reconnu le "réceptacle" du Timée. A ce moment de l'analyse, il importe de bien comprendre que le "réceptacle", ou tout autre nom qu' on lui donne, n'est pas une "hypothèse" forgée pour "expliquer la différence de l'image par rapport au modèle""l. De fait, la notion de "réceptacle", c'est-à-dire de support, est - j'espère l'avoir suffisamment montré - analytiquement contenue dans celle de

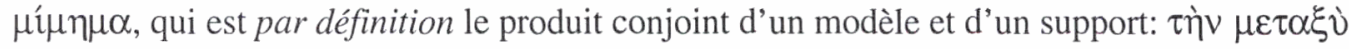

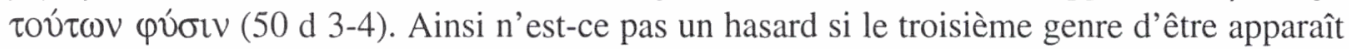
dans le Dialogue lorsque le Devenir est, pour la première fois, qualifié de $\mu$ í $\mu \eta \mu \alpha$ du Modèle. Ce n'est pas non plus un hasard si trois des quatre occurrences de $\mu$ í $\mu \eta \alpha$ dans le Timée se situent au cœur du développement sur l'v́ ciation", ce ne sera pas en tant que construction intellectuelle ou que fiction théorique. Dès lors la question ne doit plus être: pourquoi faire l'hypothèse d'un "milieu spatial” [ن́ Mais pourquoi penser la génération du Tout comme une imitation, comme une $\mu$ í $\eta_{\eta} \iota_{\varsigma}$ ? 
Il convient tout d'abord de rappeler que, de l'Ouvrier divin à l'artisan humain, fabriquer c'est imiter, c'est-à-dire faire venir à l'être une copie du modèle, qu'il s'agisse de réalités ou d' "images". Les unes et les autres seront donc, au sens strict, des $\mu \tau \eta \eta \dot{\eta} \mu \tau \tau \alpha$, et l'on voit mal comment on pourrait, sous ce seul chef, les faire toutes glisser du côté de l'irréalité. Or, pour qu'un tel processus de fabrication imitatrice ou d'imitation productive, comme on voudra, puisse se dérouler, il est nécessaire - d'une nécessité ontologique - de poser l'existence, aux côtés des formes intelligibles et des choses sensibles, d'un troisième

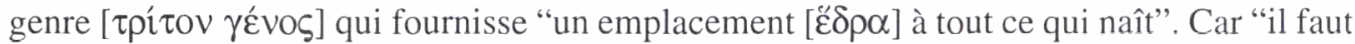

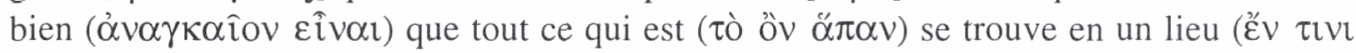

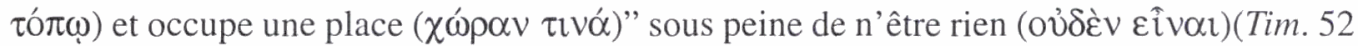

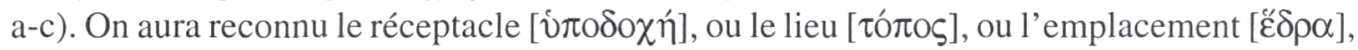

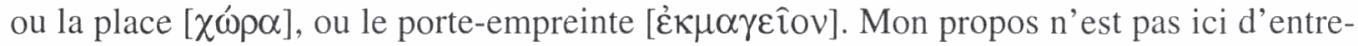
prendre l'analyse de ces différentes appellations. Je retiendrai simplement la définition qu'en

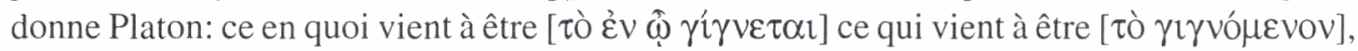
ce sans quoi aucune venue à l'être ne serait possible (Tim. 50 d). L'existence du troisième genre de réalité permet donc la transcription démiurgique du Vivant intelligible, "de façon à

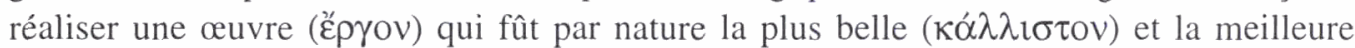

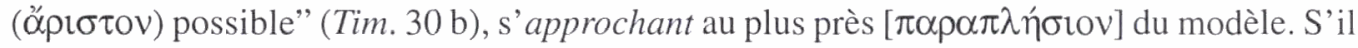

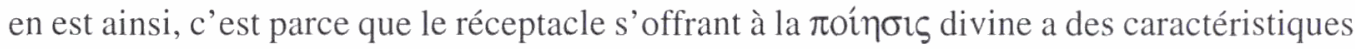
telles qu'il prend au mieux la ressemblance de ce dont il reçoit l'empreinte: il "est absolument dépourvu des formes de toutes les espèces qu' [il] est susceptible de recevoir" (Tim. 50

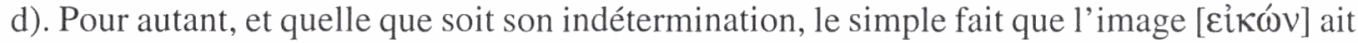
besoin du réceptacle pour "venir à l'être" et ne pas demeurer "toujours le fantôme de quel-

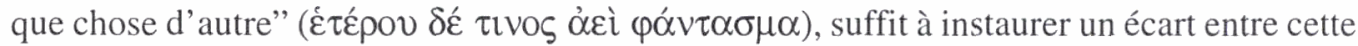

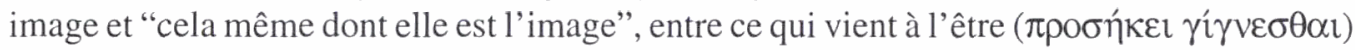

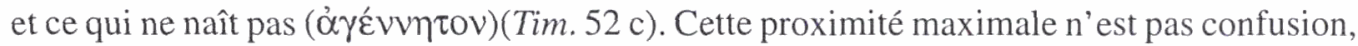

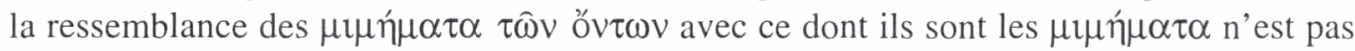
faux-semblant (Tim. 50 c). Se trouve ainsi fondée, au niveau de la démiurgie divine, la fonctionnalité propre de l'عíkóv: donner à penser la distance ontologique qui sépare le modèle de sa copie. Il en sera de même en ce qui concerne la $\pi$ oínoı h humaine, à ceci près qu'il s'agit maintenant de la transcription d'un modèle sur un support qui n'a pas les qualités de celui qu'utilise le dieu artisan et qui va imposer ses propres exigences, ses propres contraintes, à l'opération à laquelle il se prête. Inévitablement l'écart avec le modèle sur

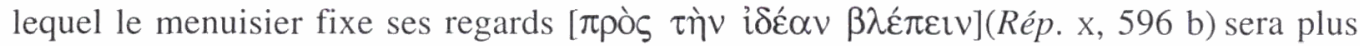
grand: le matériau, en effet, "en prend[ra] mal la ressemblance, étant donné qu'il montre[ra] en même temps l'aspect qui est le sien" (Tim. 50 e). Remarquons-le cependant, c'est cette transcription qui permet à l'objet de remplir la fonction qui est la sienne: personne n'a jamais dormi dans un Lit idéal, alors qu'il entre dans l'Eîßoৎ du lit que je puisse y dormir. En d'autres termes, l'imitation du fabricant de lits ne consiste pas uniquement à faire venir à l'être un objet nécessairement défectueux par rapport au $\pi \alpha \rho \alpha ́ \delta \varepsilon \imath \gamma \mu \alpha$ dont il est la copie. À travers lui, d'une certaine façon, le $\pi \alpha p \alpha ́ \delta \varepsilon \imath \gamma \mu \alpha$ s'accomplit, et cet accomplissement passe par son inscription dans le sensible. Victor Goldschmidt a donc raison de soutenir que "l'Univers platonicien n'est pas, comme le ressasse une tradition tenace et paresseuse, coupé en deux" (Goldschmidt, 1985, p. 112). 


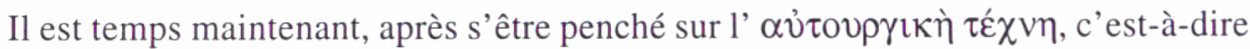

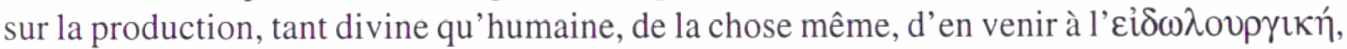
c'est-à-dire à la production de son image. L'une comme l'autre impliquent un changement de support (Soph. 266 d). La transcription du modèle - êtres naturels ou objets fabriqués - se fera sur un support autre, lequel à son tour imposera ses exigences propres. Cette fois ce n'est pas seulement le transport du modèle dans ce qui doit le recevoir qui crée l'écart avec sa copie mais, comme nous l'avons vu en analysant les attestations de

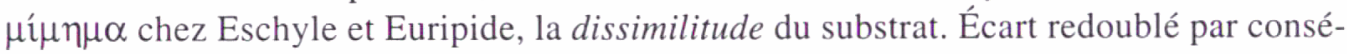
quent. Faut-il mettre l'accent exclusivement sur ce redoublement qui marque un éloignement plus grand par rapport à ce qui est réellement réel? Ou faut-il privilégier la répétition continue d'un mode de surgissement qui, du Démiurge fabricant le monde aux peintres, aux sculpteurs et aux poètes, instaure entre les différents niveaux de l'être et de l'apparaitre une connivence et des affinités que ne doivent pas masquer les pratiques déviantes aux yeux de Platon - de certains $\mu \mu \eta \eta \alpha \alpha^{\prime}$ ? L'un et l'autre, car c'est dans cette double attitude que s'enracine la rectitude de l'image. L'imitateur, en imitant, imite l'Ouvrier divin et donc, d'une certaine façon, se rend semblable au dieu autant qu'il est possible. Or on sait que c'est là tâche humaine par excellence ${ }^{23}$. Ce n'est donc pas parce $q u$ 'il imite que le $\mu \imath \mu \eta \tau$ ćs doit être chassé de la Cité, et le produit de son imitation disqualifié. Mais parce que son imitation, trompeuse dans son intention, vise à dissimuler le support qui la fait être et être ce qu'elle est. Ce qui a pour effet de faire dis-paraître l'écart entre la copie et le modèle, entre l'image et ce dont elle est l'image. Voilà le lieu d'intervention de l'expertise technicienne: dans cet art qui consiste à faire oublier que la terre cuite n'est pas chair, qu'une représentation bi-dimensionnelle ne peut rendre la tridimensionnalité de son modèle ${ }^{24}$.

\section{Notes}

* Sauf indication contraire, les traductions suivies sont celles de L. Brisson (1987), (1989), (1992); M. Canto-Sperber (1993), (1993): N. Cordero (1993); C. Dalimier (1998); M. Dixsaut (1991); M. Narcy (1994); L. Robin (1950), et, pour les autres dialogues, celles de la Collection des Universités de France. Par ailleurs, je tiens à signaler qu'une grande partie de cette communication est reprise dans les sections 3 et 4 de mon article "Idoles, icônes et phantasmes dans les Dialogues de Platon”, Revue de Métaphysique et de Morale, Juillet-Septembre 2000, n³, p. 301327.

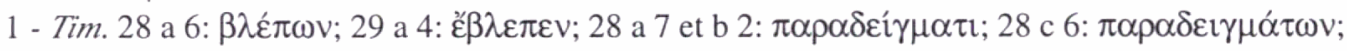

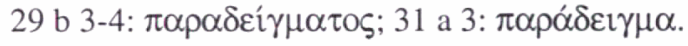

2 - Goldschmidt (1970, p. 83 n. 28). Voir également Goldschmidt (1971, § 14, p. 27 n. 8; § 72, p. 173 n. $19 ; \S 152$, p. 317 n. 2). Sur $\beta \lambda \varepsilon ́ \pi \omega, c f$. L. Paquet (1973) et A. Prévot (1935, p. 258-263).

3 - Je renverrai ici à Goldschmidt (1970 et 1985).

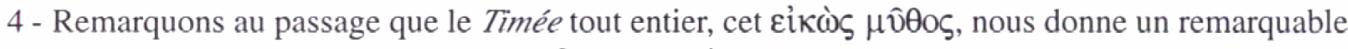
exemple des talents de ces "artisans ( $\delta \eta \mu$ tovpyoù) [habiles] à créer de belles images en discours

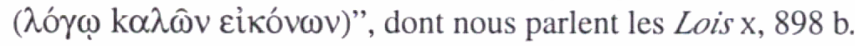

5 - Sur la notion d'intermédiarité chez Platon, cf. J. Souilhé (1919). 
6 - Sur la valeur de l'aoriste, $c f$. Humbert (1986, p. 141, § 241).

7 -Euripide, Hélène, v. 591 (Teucer); v. 875 (Ménélas). Notons que Platon, quant à lui, préfère

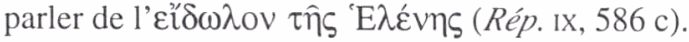

8 - Sur cette question, $c f$. Loraux (1984).

9 - Cf. l'Iphigénie à Aulis, vv. 1282-1284; Andromaque, vv. 293-301.

10 - Il. III, 16, 30, 37, 58; vI, 332, 517; XI, 581; XIII, 774.

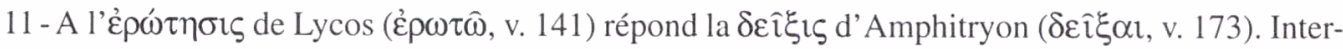
rogation-Démonstration ne forment ainsi qu'un seul et même discours.

12 - Notons de surcroît que trois des travaux d'Héraclès le mette en relation avec le monde des morts.

13 - Outre que la mort de Mégara et des Héraclides ne s'accompagne d'aucun exploit, on peut voir

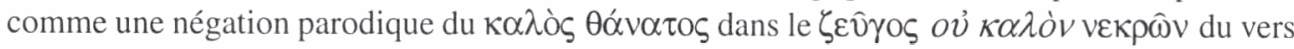
454. Il n'est pas jusqu'à l'usage de veкpós qui ne soit ici déviant: le mot, en effet s'applique "surtout [aux] soldats morts au combat". Cf. Chantraine (1980), s. v. veкpós, p. 741.

14 - Cette acceptation tient du suicide: Mégara, d'ailleurs, pour désigner sa fin, utilise le même mot qu'Héraclès pour évoquer son suicide: $\kappa \alpha \tau \theta \alpha v \varepsilon \hat{\imath} v$ (vv. 281 et 1241). Notons de surcroît que les mots pour dire le meurtre (

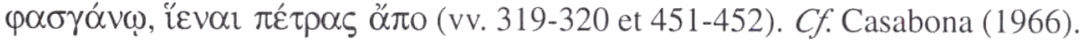

15 - On retrouve la même proximité du rire et de la honte dans le discours de Mégara: $\gamma \varepsilon ́ \lambda \omega v$, au vers 285 , et $\alpha \hat{i} \chi \chi \rho \circ \hat{\imath} \sigma \mathrm{l}$, au vers 293.

16 - Sur ce qui caractérise la mort au féminin, il faut lire Loraux (19852).

17 - Il n'est pas qu'Aristote, en effet, pour affirmer que c'est à des “modifications matérielles, c'està-dire corporelles” qu' on doit la différence sexuelle (Métaphysique I, 9, 1058 b). On sait depuis Homère, depuis Hésiode, que hommes et femmes ne sont pas issus de la même terre: "terre glaise", pour la première femme, "et non pas [...] glèbe féconde" comme pour le premier autochtone (Loraux, 1981, p. 83 et n. 36). Voir également p. 41.

18 - Héphaistos façonne les cnémides et le bouclier d'Héraclès (Hésiode, Bouclier, vv. 123 et 219); Pallas Athéna lui donne une cuirasse d'or. Par ailleurs écoutons Paul Mazon (Introduction au Bouclier dans la C. U. F., p. 126): "Certains détails de sa décoration tiennent de la magie: qui le contemple entend grincer les dents des serpents (164), sonner le bronze sous les pas des Gorgones (232); il voit frémir le feuillage de la vigne (299), ou Persée, volant à travers les airs, se détacher de la surface du bouclier (218)". C'est moi qui souligne. Sur Athéna et Héphaistos, divinités “magiciennes", cf. Detienne et Vernant (1974, p. 169-177); sur le lien Athéna-Héraclès, cf. Loraux (19812, p. 493).

19 - Phobos et Deimos encadrent la face de Gorgo sur le bouclier d'Agamemnon (Il. XI, 32), entrâ1nent le char d'Arès (Bouclier, v. 144), et ornent l'égide.

20 - Sur Créuse, il faut lire les pages 236-244 auxquelles ce développement doit beaucoup.

21 - Ce dont on fait l'hypothèse, c'est l'ensemble du schéma imitatif: modèle/support/copie, et non le seul support comme semble le penser Brisson (19742, p. 195-196). Voir aussi les pages 177 et 197.

22 - Quatre occurrences de $\mu$ í $\mu \eta \mu \alpha$ dans le Timée: 40 d 2, 49 a 1, 50 c 5, 51 b 6.

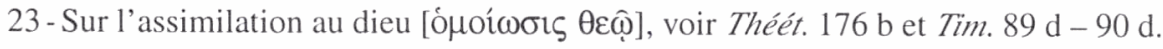

24 - Sur ce point, $c f$. Ringbom (1965, p. 102). 


\section{Bibliographie}

BENVENISTE, E. Noms d'agent et noms d'action en indo-européen. Paris: Librairie d'Amérique et d'Orient, Adrien Maisonneuve, 1975.

BRISSON, L. Le même et l'autre dans la structure ontologique du Timée de Platon. Un commentaire systématique du Timée de Platon. Paris: Klincksieck, 1974.

Platon - Lettres. Traduction inédite, introduction et notes. Paris: Flammarion, 1987.

Platon - Phèdre. Traduction inédite, introduction et notes. Paris: Flammarion, 1989.

Platon - Timée-Critias. Traduction inédite, introduction et notes. Paris: Flammarion, 1992.

Platon, les mots et les mythes. Comment et pourquoi Platon nomma le mythe? Édition revue et mise à jour. Paris: La Découverte, 1994 ( $1^{\text {re }}$ éd. 1982).

CANTO-SPERBER, M. Platon - Ménon, Traduction inédite, introduction et notes. Paris: Flammarion, 1993.

Platon - Gorgias, Traduction inédite, introduction et notes. Paris: Flammarion, $1993^{2}$.

CASABONA, J. Recherches sur le vocabulaire des sacrifices en grec, des origines à la fin de l'époque classique (Publications des Annales de la Faculté des Lettres et Sciences Humaines d'Aix, N.S. 56). Aix-en-Provence: 1966.

CHANTRAINE, P. Dictionnaire étymologique de la langue grecque. Histoire des mots. Paris: Klincksieck, 1980.

CORDERO, N. Platon - Le Sophiste. Traduction inédite, introduction et notes. Paris: Flammarion, 1993.

DALIMIER, C. Platon - Cratyle. Traduction inédite, introduction, notes, bibliographie et index. Paris: Flammarion, 1998.

DETIENNE, M. et VERNANT, J.-P. Les ruses de l'intelligence. La mètis des Grecs. Paris: Flammarion, 1974.

DIXSAUT, M. Platon - Phédon. Traduction nouvelle, introduction, notes. Paris: Flammarion, 1991.

GOLDSCHMIDT, V. "Le paradigme dans la théorie platonicienne de l'action” (1945 [1947]), repris dans Questions platoniciennes. Paris: Vrin, 1970.

. Le paradigme dans la dialectique platonicienne. Paris: Vrin-Reprise, 1985 (1éd. 1947).

HUMBERT, J. Syntaxe grecque. Paris: Klincksieck, 1986 (1 $1^{\text {re }}$ éd. 1945).

LORAUX, N. Les enfants d'Athéna. Idées athéniennes sur la citoyenneté et la division des sexes. Paris: Maspero, 1981.

. "Le héros, son bras, son destin". In: Y. Bonnefoy (dir.). Dictionnaire des mythologies. Paris: Flammarion, $1981^{2}$, t. I, p. 492-498.

. "Le fantôme de la sexualité". Nouvelle Revue de Psychanalyse, 29, 1984, p. 11-31.

"Socrate, Platon, Héraklès: sur un paradigme héroïque du philosophe”. In: J. BRUNSCHWIG, C.; IMBERT, A.; ROGER (edd.). Histoire et Structure. A la mémoire de Victor Goldschmid. Paris: Vrin, 1985, p. 93-105.

. Façons tragiques de tuer une femme. Paris: Hachette, $1985^{2}$.

NARCY, M. Platon - Théétète. Traduction inédite, introduction et notes. Paris: Flammarion, 1994. 
PAQUET, L. La médiation du regard. Essai d'interprétation. Leiden: E. J. Brill, 1973.

PRÉVOT, A. "Verbes grecs relatifs à la vision et noms de l'œil". Revue de Philologie, 1935, p. 133-279.

RINGBOM, S. "Plato on Images". Theoria, 31, 1965, p. 86-109.

ROBIN, L. Platon - CEuvres complètes, avec la collaboration de J. Moreau. Paris: Gallimard, Bibliothèque de la Pléiade, 1950.

SAÏD, S. "Deux noms de l'image en grec ancien: idole et icône". Comptes Rendus de l'Académie des Inscriptions et Belles Lettres, 1987, p. 309-330.

SOUILHÉ, J. La notion platonicienne d'intermédiaire dans la philosophie des Dialogues. Paris: Félix Alcan, 1919.

VERNANT, J.-P. "Figuration de l'invisible et catégorie psychologique du double: le colossos", dans Mythe et pensée chez les Grecs, II, 1974, p. 65-78.

. "Travail et nature dans la Grèce ancienne", dans Mythe et pensée chez les Grecs, II, $1974^{2}$, p. $16-43$

“Catégories de l'agent et de l'action en Grèce ancienne". In: J. Kristeva, J.-C.; Milner, N. Ruwet. Langues, discours, société. Pour Emile Benveniste, Paris: Seuil, 1975.

Figures, idoles, masques. Conférences, essais et leçons du Collège de France, Paris: Julliard, 1990.

VIDAL-NAQUET, P. Le Chasseur noir. Formes de pensée et formes de société dans le monde grec. Paris: Maspero, 1981.

DESCLOS, M.-L. Une façon platonicienne d'être une image: le mimema. Classica, São Paulo, 13/14, p. 205-217, 2000/2001.

ABSTRACT: The article proposes a reflection on the ontological status of image as a result, understood as mimema, of the productive process which is the demiurgic activity, understood as mimesis. The notion of mimema is analyzed through passages of Aeschylus and Euripides and understood as the transcription, rather than the reproduction, of a model on a different support. By studying the context in which the term mimema appears in the poets, the author sheds light precisely on that which will be conceptualized by Plato, in his theory of mimesis.

KEYWORDS: Mímema; mímesis; Plato. 